\title{
The development of microalbuminuria is associated with raised longitudinal adiponectin levels in female but not male adolescent patients with type 1 diabetes
}

\author{
R. Amin • J. Frystyk $\cdot$ K. Ong $\cdot$ R. N. Dalton • \\ A. Flyvbjerg $\cdot$ D. B. Dunger
}

Received: 12 February 2008 / Accepted: 30 May 2008 / Published online: 12 July 2008

(C) Springer-Verlag 2008

\begin{abstract}
Aims/hypothesis We determined the longitudinal relationship between adiponectin levels and the development of microalbuminuria in an inception cohort of children with type 1 diabetes.

Methods Blood samples collected annually over a median of 9.0 (range 1.3-14.9) years were assayed for adiponectin and $\mathrm{HbA}_{1 \mathrm{c}}$ in 55 children (36 girls) with type 1 diabetes and microalbuminuria whose age of onset of diabetes was 9.4 years (range 2.2-15.4). Samples were also assayed from normoalbuminuric children (controls) matched for age, sex and duration of diabetes.

Results Overall, adiponectin levels were higher in girls than in boys, but only after 11 years of age (median [range]: 15.3 [5.8-124.4] vs 11.6 [4.1-26.5] mg/l, $p<0.001)$. Furthermore, adiponectin levels were higher in girls with micro-
\end{abstract}

R. Amin • D. B. Dunger $(\bowtie)$

University Department of Paediatrics, Box 116,

Level 8, Addenbrooke's Hospital,

Hills Road,

Cambridge CB2 2QQ, UK

e-mail: dbd25@cam.ac.uk

J. Frystyk $\cdot$ A. Flyvbjerg

Medical Research Laboratories, Clinical Institute,

Aarhus University Hospital,

Aarhus, Denmark

K. Ong

MRC Epidemiology Unit, Institute of Medical Science,

Addenbrooke's Hospital,

Cambridge, UK

R. N. Dalton

The WellChild Laboratory, Guy's Hospital, Guy's and St Thomas' NHS Foundation Trust, London, UK albuminuria than in control girls, but this was only apparent after the onset of microalbuminuria ( $p=0.001$, adjusted for BMI, daily insulin dose, $\mathrm{HbA}_{1 \mathrm{c}}$ and age). In boys, adiponectin levels did not differ between those with microalbuminuria and controls. Further sex-related discordant associations with adiponectin levels were observed; in girls, adiponectin levels were positively related to $\mathrm{HbA}_{1 \mathrm{c}}$ levels $(r=0.2, p=0.05)$ and urine albumin excretion $(r=0.3$, $p<0.05)$ and inversely related to BMI $(r=-0.2, p<0.05)$. These associations were absent in boys.

Conclusions/interpretation In adolescent girls with type 1 diabetes but not in boys, adiponectin levels increase with increasing urine albumin excretion and onset of microalbuminuria. Although causal links cannot be inferred, this sexual dimorphism may reflect interactive effects of hyperglycaemia and sex steroids on risk of complications and adiponectin production.

Keywords Adiponectin - Adolescence - Body mass index · Gender $\cdot$ Microalbuminuria $\cdot$ Puberty $\cdot$ Type 1 diabetes

\author{
Abbreviations \\ ACR albumin/creatinine ratio \\ ORPS Oxford Regional Prospective Study \\ SDS standard deviation score
}

\section{Introduction}

In the general population, the adipocytokine adiponectin is negatively associated with visceral adiposity, fasting glucose levels and insulin resistance [1-3]. Low levels precede a decrease in insulin sensitivity [4] and are associated with obesity and the development of type 2 diabetes and 
cardiovascular disease [5]. Weight loss is associated with increased adiponectin levels [6], which subsequently predict improvements in insulin sensitivity [7] and reduced risk of type 2 diabetes [8] and cardiovascular disease [9]. Collectively these data suggest a role for adiponectin as an endogenous insulin sensitiser and in the development of insulin resistance.

In adults with type 1 diabetes, higher adiponectin levels have been observed compared with controls [10], which, paradoxically, may be associated with microalbuminuria $[11,12]$, reduced glomerular filtration rate [11, 12], overt nephropathy and retinopathy [13]. Such observations conflict with data showing associations between insulin resistance and risk of microvascular complications in type 1 diabetes [14]. It is possible that adiponectin levels may not simply reflect levels of insulin resistance in type 1 diabetes. Complex changes in adiponectin levels are seen during childhood, as levels are higher in the newborn period [15] and fall through puberty [16]. Clear sex-related differences have been identified, with higher levels in girls than in boys during early childhood [17] and puberty [18]. These age and sex-related differences are particularly pertinent to associations with microalbuminuria risk during adolescence, which is increased in relation to diabetes duration, pubertal onset and female sex [19].

In the present study, we examined longitudinal changes in adiponectin levels in relation to the development of microalbuminuria in a cohort of children recruited at diagnosis of type 1 diabetes and followed through puberty, in comparison with age-, sex- and diabetes durationmatched diabetic controls without microalbuminuria.

\section{Methods}

Participants The Oxford Regional Prospective Study (ORPS) was established in 1985 [19]. The study recruited 494 children diagnosed with type 1 diabetes at $<16$ years of age in the geographically defined region of Oxford Health Authority between 1986 and 1996; recruitment was within 3 months of diagnosis. These participants were assessed at the end of the first year from diagnosis and annually thereafter with measurements of height, weight and urine albumin/creatinine ratio (ACR) from three consecutive early morning, first-void urine specimens. Blood samples were collected for central measurement of $\mathrm{HbA}_{1 \mathrm{c}}$. Ethical approval was obtained from the district ethics committees in the region with written consent from the parents. Children were asked to assent before entering the study and to provide consent at age 16 years.

Microalbuminuria was defined as ACR $>3.5 \mathrm{mg} / \mathrm{mmol}$ in boys and $>4.0 \mathrm{mg} / \mathrm{mmol}$ in girls and $<35 \mathrm{mg} / \mathrm{mmol}$ in two out of three consecutive early morning urine collec- tions [19]. Between 1998 and 2001, 55 (36 girls) of the 494 participants recruited into ORPS had an ACR that fell within our definition of microalbuminuria and these were designated as the microalbuminuria group. Controls were selected from the remaining 431 participants who had not developed microalbuminuria by 2001 . Microalbuminuria cases were matched 1:1 with controls by age (within 1 year), sex and duration of diabetes. For each participant, annual non-fasting blood samples were collected and stored for a median of 9.0 (range 1.3-14.9) years until September 2001. All available blood samples were analysed for adiponectin levels and $\mathrm{HbA}_{1 \mathrm{c}}$ (median number of samples per patient 6 [range $2-12$, interquartile range 4-9]).

Auxology Height was measured on wall-mounted stadiometers and weight measured on electronic scales. BMI was reported as $\mathrm{kg} / \mathrm{m}^{2}$.

Albumin assay Until 1994 urine samples were stored at $-20^{\circ} \mathrm{C}$. After this time urine was stored at $-70^{\circ} \mathrm{C}$ in response to reports of the detrimental effects of storage at $-20^{\circ} \mathrm{C}$. Albumin was measured centrally by a double antibody ELISA method described previously [19]. The within- and between-assay CVs were $6 \%$ and $12 \%$ respectively.

Creatinine Creatinine was measured using a modified Jaffe method (Unimate 7; Roche Diagnostic Systems, Basel, Switzerland) on an automated spectrophotometer (Cobas Mira; Roche Diagnostic Systems). The CV was $2 \%$ at $2.2 \mathrm{mmol} / \mathrm{l}$.

$H b A_{l c}$ Glycated haemoglobin was measured centrally, initially by an electrophoretic method (Ciba Corning Diagnostics, Halstead, UK), which was replaced by HPLC (Diamat; Bio-Rad, Hemel Hempstead, UK) in 1992. The relationship between the two methods was carefully evaluated and has been described previously [19]. The within-batch CV for the HPLC method was $2.2 \%$ and $1.3 \%$ at a level of $9.8 \%$ and $10.1 \%$, respectively. The betweenbatch $\mathrm{CV}$ was $3.5 \%$ and $2.2 \%$ at $5.6 \%$ and $10.1 \%$, respectively.

Adiponectin Serum adiponectin was analysed with an inhouse time-resolved immunofluorometric assay based on commercial reagents ( $\mathrm{R} \& \mathrm{D}$ Systems, Abingdon, UK) as recently described [13]. All samples were analysed in duplicates in a final dilution of 1:206. Within-assay CVs of standards and unknown samples averaged less than 5\%. Between-assay $\mathrm{CVs}$ were estimated by repetitive analysis of a control sample diluted 1:2,500, 1:500 and 1:50, respectively. After 111 setups, between-assay CVs averaged 
$5.9 \%$ at $4.8 \mu \mathrm{g} / 1$ (final dilution $1: 2500$ ), $3.6 \%$ at $23 \mu \mathrm{g} / 1$ (final dilution $1: 500$ ) and $2.7 \%$ at $234 \mu \mathrm{g} / 1$ (final dilution 1:50). The recovery of exogenously added adiponectin to serum was $101 \pm 1 \%$ (means \pm SEM based on ten samples). Repetitive thawing and freezing of serum for up to seven cycles did not significantly alter the immunoreactive levels of adiponectin (data not shown).

Statistical methods BMI standard deviation scores (SDS) were calculated by comparison with the British 1990 Growth Reference and Cole's LMS method [20]. As excretion of urinary albumin varies greatly within individuals and tends not to be normally distributed, we averaged three results from each participant with the geometric mean. The distributions of these yearly geometric means for the whole cohort were not normally distributed and were $\log$ transformed and analysed on this scale. Adiponectin levels were also $\log$ transformed to allow parametric analysis. Other variables were normally distributed. Data were summarised as means for each patient and compared using an ANOVA model in a case-control design. Correlation between variables was measured by Pearson's correlation coefficient. Analysis of covariance was used to test interactions with sex on the relationship between adiponectin and other covariates. Linear regression analysis was used to assess associations of covariates with the $\log$ of urine ACR.

To display longitudinal changes in adiponectin levels, we used multilevel modelling software (MLwiN version 1.0 beta; Institute of Education, London, UK). This is a hierarchical regression model, allowing consideration of an individual's longitudinal data and summation by predefined groups. Variance in adiponectin levels was modelled against age or years relative to onset of microalbuminuria. Three patients had data from only two time points and this would have generated straight lines during the creation of the model. Therefore data from these three patients were omitted from the dataset during analyses on MlwiN. To recognise longitudinal adiponectin levels for each child, two levels of hierarchy in the data were used: level 1 consisting of all measurement points, and level 2 consisting of unique codes for each child. Models with the categorical explanatory variable (microalbuminuria status or sex) were built separately and graphs of the predicted values drawn from these models. Separate models were estimated for the microalbuminuria and control groups and for sex; from these we obtained a graph of the curves of mean predicted values over time in each group and SEMs. Each model was a hierarchical random-effects model, with level 1 of the data hierarchy being the measurement points nested within child as level 2, thereby allowing for repeated measurements per child. Adiponectin levels were modelled against time using linear, quadratic and cubic terms. From these parameters we drew graphs of the mean $( \pm \mathrm{SE})$ predicted values over time in each group. MlwiN was not used for other analyses of the data set, which was done using other statistical methods with SPSS version 11.5 (SPSS, Chicago, IL, USA).

Time variables, adiponectin levels and urine ACR were reported as median (range). All other data are presented as mean (SD), unless otherwise stated. A $p$ value of $<0.05$ was considered significant.

\section{Results}

Cohort characteristics In patients with microalbuminuria compared with controls, there were no differences in age at diagnosis of diabetes $(9.4$ [2.2-15.4] vs 9.8 [2.4-16.3] years, $p=0.8)$ or duration of diabetes $(8.5[1.1-15.5]$ vs 8.3 [1.1-14.4] years, $p=0.9)$. Additional comparisons are summarised in Table 1.

Adiponectin levels in relation to age For the whole cohort, adiponectin levels fell with age $(r=-0.2, p<0.001$; Fig. 1a). As no Tanner pubertal staging was available, age $>11$ years was taken as a surrogate marker for onset of puberty. Adiponectin levels were higher at ages $<11$ years $(17.5[12.5-74.5] \mathrm{mg} / \mathrm{l})$ than after age 11 years $(13.9$ [5.2$100.5] ; p<0.001)$. The higher adiponectin levels in participants with microalbuminuria compared with controls became more apparent with chronological age (Fig. 1b,c) and were only apparent after the onset of microalbuminuria (Fig. 2).

Modification of adiponectin associations by sex Adiponectin levels were higher in girls than boys, but only after age 11 years (15.3 [5.8-124.4] vs 11.6 [4.1-26.5] mg/l, $p<0.001$; Fig. 1a). Girls also had slightly higher BMI SDS than boys $(0.7[0.9]$ vs 0.6 [0.9], $p=0.05)$, but the differences in adiponectin persisted after further adjustment for BMI SDS $(p<0.001)$.

The higher adiponectin levels in participants with microalbuminuria compared with controls was more clearly evident in girls $(p<0.001)$ than in boys $(p=0.02$; Table 1 , Fig. $1 \mathrm{~b}, \mathrm{c})$. Similarly, adiponectin levels were positively related to urine ACR in girls $(p<0.05)$, but not in boys ( $p=$ $0.71, p=0.02$ for interaction with sex; Table 2). Further associations with adiponectin levels were significantly discordant between sexes (Table 2); in girls, but not in boys, adiponectin levels were positively related to $\mathrm{HbA}_{1 \mathrm{c}}$ levels (Fig. 3) and were inversely related to BMI SDS. However, no discordant correlation with daily insulin dose was seen in either of the sexes $(p=0.5$ for interaction with sex; Table 2). 
Table 1 Comparison of data for participants with microalbuminuria (MA) vs controls for the whole cohort (overall), and for girls and boys considered separately
Values are median (range) (urine ACR and adiponectin) or mean (SD)

\begin{tabular}{|c|c|c|c|}
\hline Variable & MA & No MA & $p$ value \\
\hline Overall & $n=55$ & $n=55$ & \\
\hline BMI SDS & $0.6(0.9)$ & $0.7(1.0)$ & 0.7 \\
\hline Insulin dose $\left(\mathrm{U} \mathrm{kg}^{-1} \mathrm{day}^{-1}\right)$ & $0.9(0.3)$ & $0.9(0.3)$ & 0.8 \\
\hline Mean $\mathrm{HbA}_{1 \mathrm{c}}(\%)$ & $10.8(2.1)$ & $9.9(1.8)$ & $<0.001$ \\
\hline Mean urine ACR $(\mathrm{mg} / \mathrm{mmol})$ & $9.1(3.3-160.7)$ & $0.8(0.1-3.3)$ & $<0.001$ \\
\hline Mean adiponectin (mg/l) & $14.9(4.1-129.4)$ & $13.2(6.5-34.0)$ & $<0.001$ \\
\hline Girls & $n=36$ & $n=36$ & \\
\hline BMI SDS & $0.7(1.0)$ & $0.8(0.8)$ & 0.2 \\
\hline Insulin dose $\left(\mathrm{U} \mathrm{kg}^{-1} \mathrm{day}^{-1}\right)$ & $0.9(0.2)$ & $0.9(0.3)$ & 0.7 \\
\hline Mean $\mathrm{HbA}_{1 \mathrm{c}}(\%)$ & $10.9(0.2)$ & $10.0(0.2)$ & $<0.001$ \\
\hline Mean urine ACR $(\mathrm{mg} / \mathrm{mmol})$ & $3.6(3.5-161.8)$ & $0.9(0.1-3.4)$ & $<0.001$ \\
\hline Mean adiponectin (mg/l) & $17.5(7.0-124.4)$ & $14.5(6.1-34.6)$ & 0.006 \\
\hline Boys & $n=19$ & $n=19$ & \\
\hline BMI SDS & $0.6(1.0)$ & $0.6(0.9)$ & 0.9 \\
\hline Insulin dose $\left(\mathrm{U} \mathrm{kg}^{-1} \mathrm{day}^{-1}\right)$ & $0.9(0.3)$ & $0.8(0.3)$ & 0.5 \\
\hline Mean $\mathrm{HbA}_{1 \mathrm{c}}(\%)$ & $10.6(0.2)$ & $9.9(0.1)$ & 0.005 \\
\hline Mean urine ACR $(\mathrm{mg} / \mathrm{mmol})$ & $3.7(3.5-106.6)$ & $0.7(0.2-3.1)$ & $<0.001$ \\
\hline Mean adiponectin $(\mathrm{mg} / \mathrm{l})$ & $12.6(4.4-28.0)$ & $11.4(6.7-23.2)$ & 0.2 \\
\hline
\end{tabular}

Adiponectin levels in relation to microalbuminuria status In participants with microalbuminuria compared with controls, mean $\mathrm{HbA}_{1 \mathrm{c}}$ levels were higher (Table 1). As microalbuminuria cases were matched to controls by age and duration of diabetes, adiponectin levels in controls were related to the timing of development of microalbuminuria in each matched case and analysed by years relative to onset of microalbuminuria. Adiponectin levels were higher in participants with microalbuminuria than in controls, but this was apparent only after the appearance of microalbuminuria (16.4 [5.7-100.7] vs 12.1 [5.8-34.0] mg/l, $p=$ 0.008 ; Fig. 2). These differences were not confounded by BMI SDS or daily insulin dose.

Girls with microalbuminuria had significantly higher adiponectin levels than control girls (Table 1, Fig. 1b), but this difference was not observed in boys (Table 1, Fig. 1c). Analysis by interaction with sex further confirmed the difference between the sexes $(p<0.001$ for interaction with sex). In girls this observed difference in adiponectin levels persisted after adjustment for BMI SDS, daily insulin dose, $\mathrm{HbA}_{1 \mathrm{c}}$ and age $(F=11.9, p=0.001)$.

Regression analysis revealed that in girls only increasing urine ACR was weakly associated with higher ( $\log )$ adiponectin levels $(F=3.3, p=0.07)$, after adjustment for BMI SDS, daily insulin dose, $\mathrm{HbA}_{1 \mathrm{c}}$ and age. These associations were not observed in boys.

\section{Discussion}

We provide the first description of longitudinal changes in adiponectin levels in an inception cohort of children with type 1 diabetes in relation to the development of microalbuminuria. Earlier studies have shown higher adiponectin levels in adolescents with type 1 diabetes than in nondiabetic control participants, as well as a link between increased adiponectin levels and microalbuminuria [10]. Our study shows that these observations are modified by sex and age. Adiponectin levels were higher overall in participants with microalbuminuria compared with controls, but this difference was only apparent in girls and only became evident after the onset of microalbuminuria.

Increased adiponectin levels predict improvements in insulin sensitivity [7], suggesting a role for adiponectin as an endogenous insulin sensitiser. Observations of raised adiponectin levels in relation to increased urine albumin excretion in girls but not in boys in our study may be important as, in addition to insulin resistance, factors influencing adiponectin levels such as age [15, 16], sex [17] and puberty [18] are also associated with risk of microalbuminuria during adolescence [19].

Previous cross-sectional studies in adults with type 1 diabetes have shown a variable relationship between adiponectin levels and glycaemic control, with a negative [1], a positive [11] or no correlation at all [13]. In these studies, differences between the sexes were either not found or not reported. Our study is the first to show a sexual dimorphism in the relationship of adiponectin and $\mathrm{HbA}_{1 \mathrm{c}}$ levels in type 1 diabetes. The sex-related difference may be relevant to risk of microalbuminuria, as this is strongly associated with poor glycaemic control, with female adolescents generally having higher $\mathrm{HbA}_{1 \mathrm{c}}$ levels than males [19]. Although the mechanism is unknown, experimental data have shown that exposure of adipocytes to hyperglycaemia results in post- 


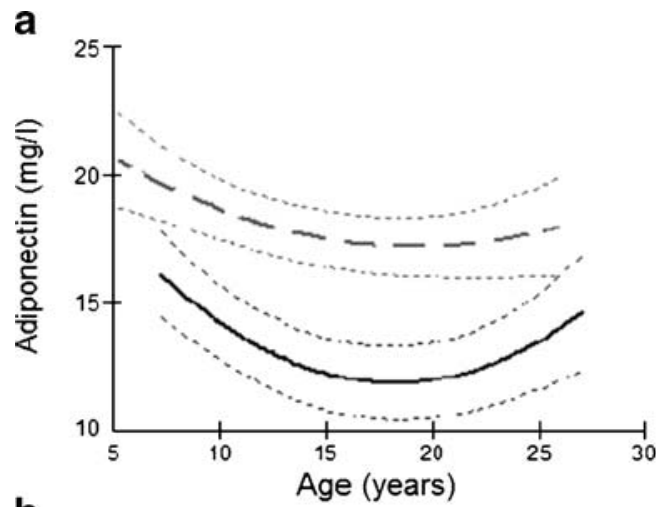

b
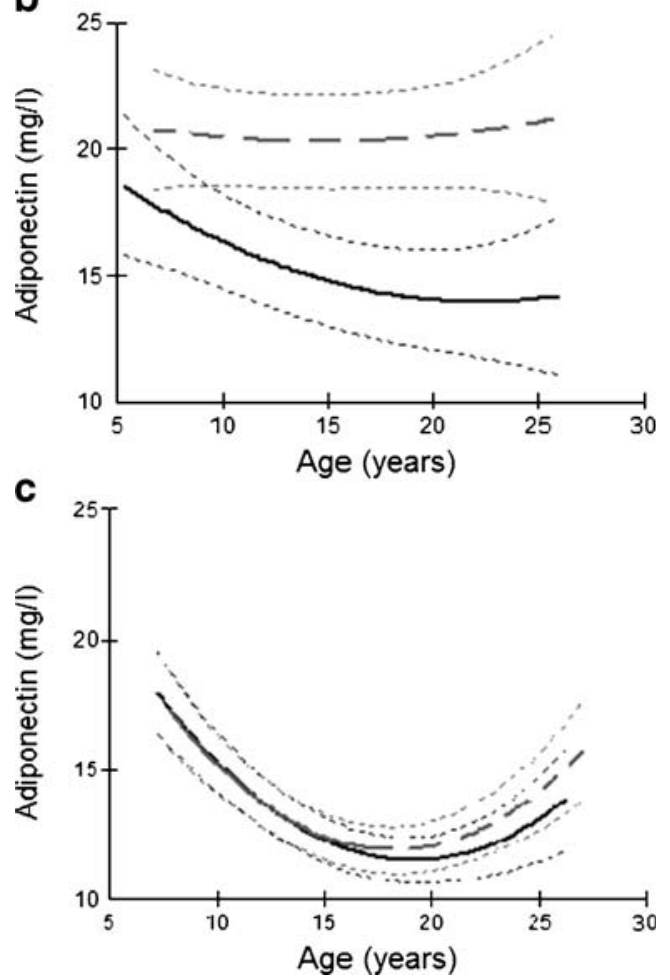

Fig. 1 Adiponectin levels plotted across age (a) in relation to sex in patients with and without microalbuminuria $(p<0.001$; solid line, male; dashed line, female), (b) in female microalbuminuria patients (dashed line) and controls (solid line; $p=0.006$ ) and (c) in male microalbuminuria cases (dashed line) and controls (solid line; $p=0.2$ ). Graphs were derived from multilevel modelling (MLwiN version 1.0 beta). Dotted lines: \pm SEM

translational modification of adiponectin [21], which may decrease its action in the liver [22]. Therefore, it is possible that the higher adiponectin levels in female participants with microalbuminuria reflect a compensatory increase in secretion of adiponectin due to chronic hyperglyceamic exposure and reduced action of modified adiponectin at the liver.

Our data also show that subcutaneous insulin dose was inversely associated with adiponectin levels in girls but not in boys, a finding that is consistent with studies of adults with type 1 diabetes [23]. In diabetic rodent models, low

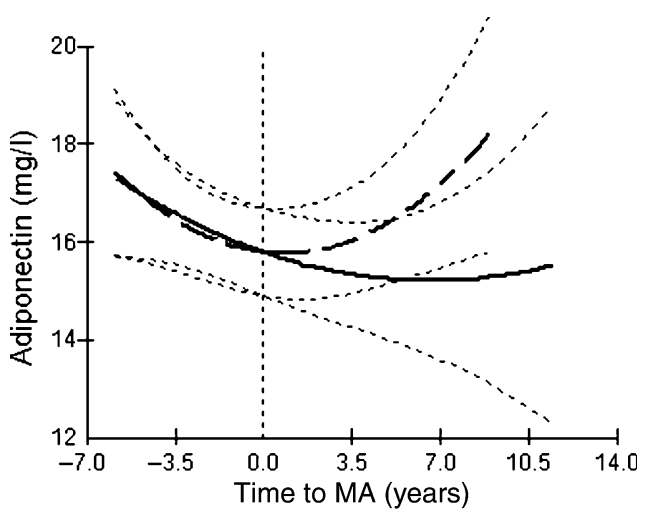

Fig. 2 Differences in adiponectin levels in participants with microalbuminuria (MA; dashed line) compared with controls (solid line) in relation to the onset of microalbuminuria $(p=0.008)$. As microalbuminuria cases were matched to controls by age and duration of diabetes, adiponectin levels in controls were related to timing of development of microalbuminuria in each matched case and plotted years are relative to onset of microalbuminuria. Graph derived from multilevel modelling (MLwiN version 1.0 beta). Dotted lines: \pm SEM

adiponectin levels increased initially with insulin treatment [24]. These inconsistent findings require further study.

In the general population, girls develop higher adiponectin levels than boys through childhood and show an inverse association with BMI, while in boys this association remains positive [17]. Although unproven, this sex-related difference in association between adiponectin and adiposity may relate to the timing of accumulation of intra-abdominal fat and greater insulin resistance in females than in males [25] and to the effects of sex steroids [18]. In type 1 diabetes, timing of puberty is altered [26] and BMI and insulin resistance are increased during adolescence, particularly in girls $[27,28]$. Furthermore, risk of microalbuminuria in female adolescents is associated with higher androgen levels [29]. This may relate to the increased frequency of ovarian hyperandrogenism, which may be present in up to $50 \%$ of adolescent girls with type 1 diabetes [30] and is associated with obesity, insulin resistance and peripheral hyperinsulinaemia [31]. Collectively these factors may contribute to the sexual dimorphism in adiponectin levels.

Given the role of adiponectin as an endogenous insulin sensitiser [4, 6, 7] and its anti-atherogenic effects [9], associations between increased adiponectin levels and risk of microalbuminuria in females, which has been linked to insulin resistance [28], is paradoxical. The observations may reflect a compensatory attempt to mitigate generalised microvascular damage by improving insulin sensitivity. This is speculative, but recent data have shown a correlation between adiponectin levels and both TNF- $\alpha$ levels and the soluble vascular cell adhesion molecule- 1 (sVCAM-1). These are markers of generalised endothelial 
Table 2 Discordant correlation between adiponectin levels and other variables in girls and boys

\begin{tabular}{lcccc}
\hline Determinant & Both sexes & Girls & Boys & $p$ value for interaction with sex \\
\hline Daily insulin dose & $-0.2^{* * *}$ & $-0.2^{*}$ & -0.1 & 0.5 \\
BMI SDS & $-0.2^{* * *}$ & $-0.2^{*}$ & -0.04 & 0.01 \\
$\mathrm{HbA}_{1 \mathrm{c}}$ & $0.2^{*}$ & $0.2^{*}$ & -0.1 & 0.004 \\
Urine ACR & $0.2^{*}$ & $0.3^{*}$ & 0.1 & 0.02 \\
\hline
\end{tabular}

${ }^{*} p<0.05, * * * p<0.001$

dysfunction [12] and indicate that adiponectin is linked to inflammation, atherosclerosis and endothelial damage. Our data may be confounded by differences between males and females in production of various isoforms of adiponectin, which current assays do not measure. Females produce higher levels of the high molecular mass isoform, which is known to differ in its receptor binding properties and metabolic effects [32]. Alternatively there may be sexrelated differences in genetic regulation of adiponectin expression [33]. However, other data show that polymorphisms of the adiponectin gene are not associated with nephropathy in adults with type 1 diabetes [34]. A limitation of our study is that the discordant sex-related associations in adiponectin and $\mathrm{HbA}_{1 \mathrm{c}}$ levels and urine albumin excretion may be related to the smaller sample size in boys than in girls, and our study may not have been powered to detect sex-related differences. However, testing for interaction with sex supported these discordant observations. In addition, higher adiponectin levels in adults have been found in association with reduced glomerular filtration rate, suggesting reduced clearance of adiponectin $[11,12]$.

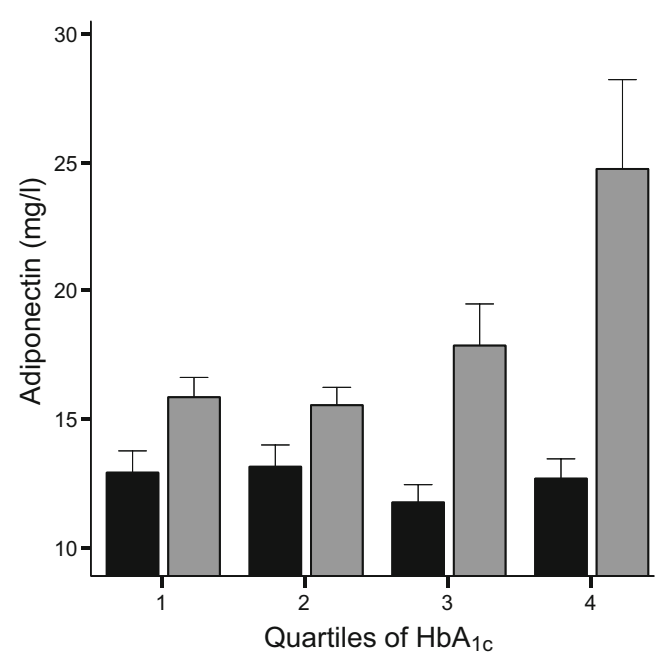

Fig. 3 Adiponectin levels in male (black bars) and female (grey bars) type 1 diabetic participants, plotted against quartiles of $\mathrm{HbA}_{1 \mathrm{c}}(p<$ 0.001 for difference in trends between male and female participants). Errors bars are SEM
In summary, adiponectin levels increase with the onset of microalbuminuria, but these changes are restricted to females. Although causal links cannot be inferred, these findings may relate to chronic hyperglycaemia, accumulation of intra-abdominal fat mass, insulin resistance and the increased risk of microvascular disease in pubertal girls with type 1 diabetes.

Acknowledgements The Oxford Regional Prospective Study is funded by Diabetes UK. We acknowledge the Novo Nordisk UK Research Foundation, which has previously sponsored R. Amin, the Juvenile Diabetes Research Foundation and the WellChild Trust. In addition this study was supported by grants from the Danish Research Council for Health and Disease and the Danish Diabetes Association. Thanks are also due to the study field workers, A. Watts and D. Harris for laboratory assistance, the Barts-Oxford Study field workers, and the paediatricians, physicians and diabetes nurse specialists in the Oxford Region.

Duality of interest The authors declare that there is no duality of interest associated with this manuscript.

\section{Appendix}

Members of the Oxford Regional Prospective Study Steering Committee are: D. B. Dunger, R. N. Dalton, J. Fuller, E. A. M. Gale, H. Keen, M. Murphy, H. A. W. Neil, C. J. Schultz, R. J. Young and T. Konopelska-Bahu. Members of the Oxford Regional Prospective Study are: J. A. Edge, John Radcliffe Hospital Oxford; H. A. W Niel and D. Matthews, The Oxford Centre for Diabetes, Endocrinology and Metabolism, The Churchill Hospital, Oxford; R. A. F. Bell and A. Taylor, Horton General Hospital, Banbury; A. Mukhtar, B. P. O’Malley, B. R. Silk and E. H. Smith, Kettering District Hospital, Kettering; R. D. M. Scott, King Edward VII Hospital, Windsor; F. M. Ackland, C. J. Fox and N. K. Griffin, Northampton General Hospital; N. Mann, H. Simpson, P. Cove Smith and M. Pollitzer, Royal Berkshire Hospital, Reading; R. S. Brown and A. H. Knight, Stoke Mandeville Hospital, Aylesbury; J. M. Cowen and J. C. Pearce, Wexham Park Hospital, Slough. 


\section{References}

1. Fernandez-Real JM, Botas-Cervero P, Lopez-Bermano A et al (2004) Adiponectin is independently associated with glycosylated haemoglobin. Eur J Endocrinol 150:201-205

2. Yatagai T, Nagasaka S, Taniguchi A et al (2003) Hypoadiponectinemia is associated with visceral fat accumulation and insulin resistance in Japanese men with type 2 diabetes mellitus. Metabolism 52:1274-1278

3. Ducluzeau PH, Cousin P, Malvoisin E et al (2003) Glucose-toinsulin ratio rather than sex hormone-binding globulin and adiponectin levels is the best predictor of insulin resistance in nonobese women with polycystic ovary syndrome. J Clin Endocrinol Metab 88:3626-3631

4. Stefan N, Vozarova B, Funahashi T et al (2002) Plasma adiponectin concentration is associated with skeletal muscle insulin receptor tyrosine phosphorylation, and low plasma concentration precedes a decrease in whole-body insulin sensitivity in humans. Diabetes 51:1884-1888

5. Cote M, Mauriege P, Bergeron J et al (2005) Adiponectinemia in visceral obesity: impact on glucose tolerance and plasma lipoprotein and lipid levels in men. J Clin Endocrinol Metab 90:1434-1439

6. Kopp HP, Krzyzanowska K, Mohlig M, Spranger J, Pfeiffer AF, Schernthaner G (2005) Effects of marked weight loss on plasma levels of adiponectin, markers of chronic subclinical inflammation and insulin resistance in morbidly obese women. Int J Obes Relat Metab Disord 29:766-771

7. Tschritter O, Fritsche A, Thamer C et al (2003) Plasma adiponectin concentrations predict insulin sensitivity of both glucose and lipid metabolism. Diabetes 52:239-243

8. Kanaya AM, Harris T, Goodpaster BH, Tylavsky F, Cummings SR (2004) Adipocytokines attenuate the association between visceral adiposity and diabetes in older adults. Diabetes Care 27:1375-1380

9. Pischon T, Girman CJ, Hotamisligil GS, Rifai N, Hu FB, Rimm EB (2004) Plasma adiponectin levels and risk of myocardial infarction in men. JAMA 291:1730-1737

10. Galler A, Gelbrich G, Kratzsch J, Noack N, Kapellen T, Kiess W (2007) Elevated serum levels of adiponectin in children, adolescents and young adults with type 1 diabetes and the impact of age, gender, body mass index and metabolic control: a longitudinal study. Eur J Endocrinol 157:481-489

11. Saraheimo M, Forsblom C, Fagerudd J et al (2005) Serum adiponectin is increased in type 1 diabetic patients with nephropathy. Diabetes Care 28:1410-1414

12. Schalkwijk CG, Chaturvedi N, Schram MT, Fuller JH, Stehouwer CD (2006) Adiponectin is inversely associated with renal function in type 1 diabetic patients. J Clin Endocrinol Metab 91:129-135

13. Frystyk J, Tarnow L, Krarup Hansen T, Parving HH, Flyvbjerg A (2005) Increased serum adiponectin levels in type 1 diabetic patients with microvascular complications. Diabetologia 48:1911-1918

14. Yip J, Mattock MB, Morocutti A, Sethi M, Trevisan R, Viberti G (1993) Insulin resistance in insulin-dependent diabetic patients with microalbuminuria. Lancet 342:883-887

15. Kotani Y, Yokota I, Kitamura S, Matsuda J, Naito E, Kuroda Y (2004) Plasma adiponectin levels in newborns are higher than those in adults and positively correlated with birth weight. Clin Endocrinol (Oxf) 61:418-423

16. Cianfarani S, Martinez C, Maiorana A, Scire G, Spadoni GL, Boemi S (2004) Adiponectin levels are reduced in children born small for gestational age and are inversely related to postnatal catch-up growth. J Clin Endocrinol Metab 89:1346-1351

17. Ong KK, Frystyk J, Flyvbjerg A, Petry CJ, Ness A, Dunger DB (2006) Sex-discordant associations with adiponectin levels and lipid profiles in children. Diabetes 55:1337-1341
18. Bottner A, Kratzsch J, Muller G et al (2004) Gender differences of adiponectin levels develop during the progression of puberty and are related to serum androgen levels. J Clin Endocrinol Metab 89:4053-4061

19. Schultz CJ, Konopelska-Bahu T, Dalton RN et al (1999) Microalbuminuria prevalence varies with age, sex, and puberty in children with type 1 diabetes followed from diagnosis in a longitudinal study. Oxford Regional Prospective Study Group. Diabetes Care 22:495-502

20. Cole TJ (1990) The LMS method for constructing normalized growth standards. Eur J Clin Nutr 44:45-60

21. Richards AA, Stephens T, Charlton HK et al (2006) Adiponectin multimerization is dependent on conserved lysines in the collagenous domain: evidence for regulation of multimerization by alterations in posttranslational modifications. Mol Endocrinol 20:1673-1687

22. Wang Y, Lam KS, Chan L et al (2006) Post-translational modifications of the four conserved lysine residues within the collagenous domain of adiponectin are required for the formation of its high molecular weight oligomeric complex. J Biol Chem 281:16391-16400

23. Maahs DM, Ogden LG, Snell-Bergeon JK et al (2007) Determinants of serum adiponectin in persons with and without type 1 diabetes. Am J Epidemiol 166:731-740

24. Thule PM, Campbell AG, Kleinhenz DJ et al (2006) Hepatic insulin gene therapy prevents deterioration of vascular function and improves adipocytokine profile in STZ-diabetic rats. Am J Physiol Endocrinol Metab 290:E114-E122

25. Caprio S (1999) Relationship between abdominal visceral fat and metabolic risk factors in obese adolescents. Am J Human Biol 11:259-266

26. Ahmed ML, Connors MH, Drayer NM, Jones JS, Dunger DB (1998) Pubertal growth in IDDM is determined by HbA1c levels, sex, and bone age. Diabetes Care 21:831-835

27. Lebl J, Schober E, Zidek T et al (2003) Growth data in large series of 587 children and adolescents with type 1 diabetes mellitus. Endocr Regul 37:153-161

28. Amin R, Williams RM, Frystyk $J$ et al (2005) Increasing urine albumin excretion is associated with growth hormone hypersecretion and reduced clearance of insulin in adolescents and young adults with type 1 diabetes: The Oxford Regional Prospective Study. Clin Endocrinol (Oxf) 62:137-144

29. Amin R, Schultz C, Ong K et al (2003) Low IGF-I and elevated testosterone during puberty in subjects with type 1 diabetes developing microalbuminuria in comparison to normoalbuminuric control subjects: The Oxford Regional Prospective Study. Diabetes Care 26:1456-1461

30. Adcock CJ, Perry LA, Lindsell DR et al (1994) Menstrual irregularities are more common in adolescents with type 1 diabetes: association with poor glycaemic control and weight gain. Diabet Med 11:465-470

31. Dunaif A, Thomas A (2001) Current concepts in the polycystic ovary syndrome. Annu Rev Med 52:401-419

32. Waki H, Yamauchi T, Kamon J et al (2003) Impaired multimerization of human adiponectin mutants associated with diabetes. Molecular structure and multimer formation of adiponectin. J Biol Chem 278:40352-40363

33. Kovac IP, Havlik RJ, Foley D et al (2007) Linkage and association analyses of type 2 diabetes/impaired glucose metabolism and adiponectin serum levels in Japanese Americans from Hawaii. Diabetes 56:537-540

34. Ma J, Mollsten A, Falhammar H et al (2007) Genetic association analysis of the adiponectin polymorphisms in type 1 diabetes with and without diabetic nephropathy. J Diabetes Complications $21: 28-33$ 